\title{
Comparison of Multiscale Entropy Techniques for Lung Sound Classification
}

\author{
Achmad Rizal $^{1}$, Risanuri Hidayat ${ }^{2}$, Hanung Adi Nugroho ${ }^{3}$ \\ 1,2,3 Department of Electrical Engineering \& Information Technology, \\ Universitas Gadjah Mada, Jl Grafika no 2, Mlati, Sleman, D.I. Yogyakarta, Indonesia \\ ${ }^{1}$ School of Electrical Engineering, Telkom University \\ Jl Telekomunikasi no 1, Ters. Buah Batu, Bojong Soang, Bandung, Indonesia
}

\section{Article Info \\ Article history: \\ Received Apr 27, 2018 \\ Revised Aug 24, 2018 \\ Accepted Oct 8, 2018}

Keywords:

Multiscale entropy

Lung sound

Coarse-grained procedure

Multilayer perceptron

\begin{abstract}
Lung sound is a biological signal that can be used to determine the health level of the respiratory tract. Various digital signal processing techniques have been developed for automatic classification of lung sounds. Entropy is one of the parameters used to measure the biomedical signal complexity. Multiscale entropy is introduced to measure the entropy of a signal at a particular scale range. Over time, various multiscale entropy techniques have been proposed to measure the complexity of biological signals and other physical signals. In this paper, some multiscale entropy techniques for lung sound classification are compared. The result of the comparison indicates that the Multiscale Permutation Entropy (MPE) produces the highest accuracy of $97.98 \%$ for five lung sound datasets. The result was achieved for the scale 1-10 producing ten features for each lung sound data. This result is better than other seven entropies. Multiscale entropy analysis can improve the accuracy of lung sound classification without requiring any features other than entropy.
\end{abstract}

Copyright (C) 2018 Institute of Advanced Engineering and Science. All rights reserved.

Corresponding Author:

Achmad Rizal,

Department of Electrical Engineering \& Information Technology,

Universitas Gadjah Mada,

Jl Grafika no 2, Mlati, Sleman, D.I. Yogyakarta, Indonesia.

Email: rizal.s3te14@mail.ugm.ac.id

\section{INTRODUCTION}

Lung sound is one of the biological signals that emerge from the respiration process. Any changes in it are generated from foreign bodies or physiological changes in the respiratory tract caused by diseases [1]. Differences in patterns of lung sounds can be heard by a doctor using a stethoscope to diagnose diseases [2]. Auscultation technique, on the other hand, is very subjective for being dependent upon existing experience and expertise of the doctor.

Various techniques for analyzing lung sounds using computers have been developed. Some of these techniques include time-domain analysis techniques, such as statistical analysis based on Hjorth descriptor [3], empirical mode decomposition (EMD) [4], or fractal analysis [5]. Several researchers proposed to perform lung sound analysis in the frequency domain, such as quantile vector frequency [6] or MFCC [7]. Meanwhile, wavelet analysis has been used in [8] for classifying abnormal lung sounds.

One of the popular biological signal analysis methods is multiscale entropy (MSE), proposed by Costa et al. with a coarse-grained procedure for multiscale process and sample entropy for entropy measurement [9]. Subsequently, several variants of MSE emerge such as refined-MSE, composite-MSE or adaptive MSE (AME) [10]. Other researchers modified their entropy measurement techniques, which result in multiscale permutation entropy (MPE) [11], multiscale approximate entropy (MApEN) [12], and multiscale fuzzy entropy [13]. In the case of lung sounds, multiscale entropy has been used to analyze the 
lung sounds of pulmonary alveolitis patients [14]. The results indicated that MSE produces more consistent features compared to the spectral method. Similar to biological signal analysis, wavelet entropy is used for transformer design [15], while fault diagnosis was analyzed using sample entropy [16] and approximate entropy [17].

The performance comparison of multiscale entropy for various entropy measurement techniques has never previously been done. In this study, we compared the use of multiscale entropy for various entropy measurement techniques for lung sound classification. Entropy measurement method in this study included Shannon entropy [18], spectral entropy [18], Renyi entropy [19], wavelet entropy [20], approximate entropy [21], sample entropy [22], permutation entropy [23], and Tsallis entropy [24]. Those eighth entropies measurements are a representation of two types entropy measurement techniques: spectral entropy and embedding entropy. Embedding entropy was calculated directly from the signal in the time domain, and the spectral entropy was calculated from the signal amplitude in the frequency domain. By this research, we obtained the best multiscale entropy techniques for automatic lung sound classification. Next, we could recommend the best method for automatic lung sound analysis. From our experiment, MPE produced the highest accuracy among those eight entropies.

This paper is organized as follows. Section 2 explains the experimental method, the lung sound data used in this study, entropy measurement methods and multiscale entropy techniques. Meanwhile, the results and analysis of the test results described in Section 3. Section 4 contains the conclusions of this paper.

\section{MATERIAL AND METHODS}

Figure 1 presents the method in this paper. We here used five classes of lung sound data as the input data. Meanwhile, in the classification stage, we used multilayer perceptron and three-fold cross-validation. The following subsections explain the data, entropy measurement, and classification method in detail.

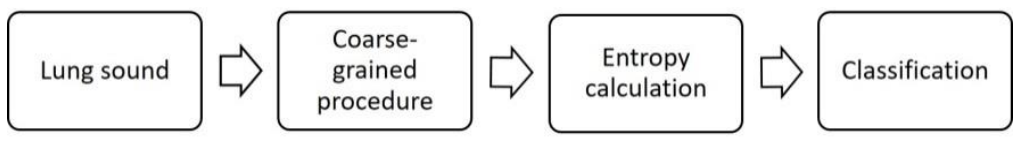

Figure 1. Block diagram of multiscale entropy in this paper

\subsection{Lung sound data}

The lung sounds were gathered from several sources on the internet $[25,26]$ and CD companion of textbook [27]. All the data were converted in the form of wave files with a sampling frequency of $8000 \mathrm{~Hz}$. Furthermore, the data were cut into one respiratory cycle.

Table 1 presents the detail of lung sound data. Some of the data have been used in a previous study [3]. Normal bronchial is a kind of normal lung sound with an expiratory duration relatively longer that inspiration phase. It has loud and high-pitch sound with a pause between inspiration and expiration [28]. Crackle is a nonmusical and explosive sound that has a short duration. Crackle sound indicates secretion as in chronic bronchitis (coarse crackle) or not correlated with secretion as in congestive heart failure (fine crackle) [28]. Asthma is a disease that produces a wheezing sound that has a continuous pattern, musical sound and dominant frequency more than $400 \mathrm{~Hz}$ [29]. Friction rub or pleural rub is associated with pleural inflammation or pleural tumors that produce nonmusical, explosive and usually biphasic sound [28]. Sometimes, it does not need a stethoscope to listen to the stridor, originating from the larynx or trachea and having a dominant frequency $>1000 \mathrm{~Hz}[8]$.

Table 1. Lung sound data

\begin{tabular}{ll}
\hline \multicolumn{1}{c}{ Data class } & Number of data \\
\hline Normal bronchial & 22 \\
Crackle & 21 \\
Asthma & 18 \\
Friction rub & 18 \\
Stridor & 20 \\
\hline
\end{tabular}

On the data, the normalization process is carried out as in (1) and (2). 


$$
\begin{aligned}
& y(n)=x(n)-\frac{1}{N} \sum_{i=1}^{N} x(i) \\
& y(n)=\frac{x(n)}{\sqrt{\frac{1}{N} \sum_{1}^{N}\left(x_{i}-\mu\right)^{2}}} \text { with } \mu=\frac{1}{N} \sum_{i=1}^{N} x(i)
\end{aligned}
$$

where $\mathrm{x}(\mathrm{n})$ is the input signal, $\mathrm{N}$ is the length of the input signal, and $\mu$ refers to the mean of the input signal. Because in (1) mean $(\mu)$ has been made as zero, so (2) can be rewritten as (3).

$$
y(n)=\frac{x(n)}{\sqrt{\frac{1}{N} \sum_{1}^{N}\left(x_{i}\right)^{2}}}
$$

The result from (3) is the mean of signal $(\mu)=0$ and standard deviation $(\sigma)=1$. The value of $\sigma=1$ would be used in the calculation of sample entropy and approximate entropy.

Figure 2 shows an example of normal bronchial and its lung sound spectrum frequency, while Figure 3 displays the crackle sound example. From Figure 2 and Figure 3, we can see the difference between the two types of signals in the time domain and the frequency domain. This is to be distinguished using multiscale entropy both spectral entropy and embedded entropy.
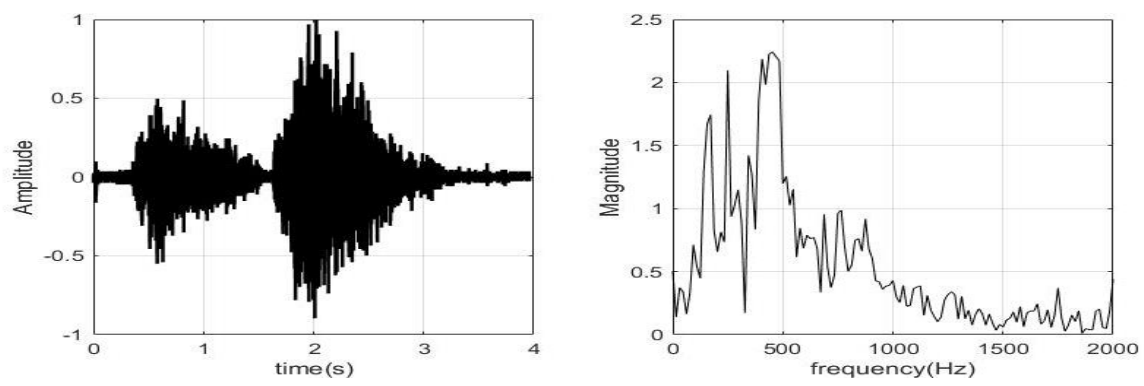

Figure 2. Normal lung sound and the spectrum
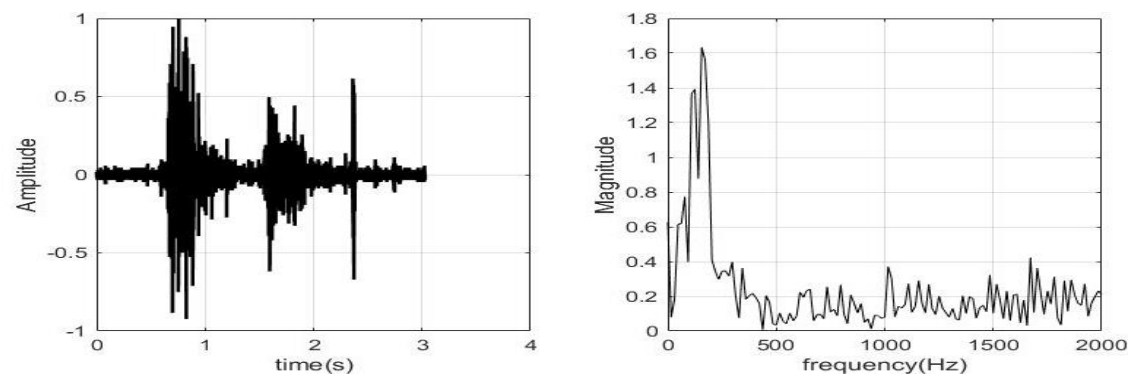

Figure 3. Crackle lung sound and the spectrum

\subsection{Multiscale process}

Multiscale entropy was used to view the physiology signal's entropy at various scales [9]. We used the coarse-grained procedure for the multiscale process as in (4).

$$
y_{j}^{(\tau)}=\frac{1}{\tau} \sum_{i=(j-1) \tau+1}^{j \tau} x_{i}, 1 \leq j \leq \frac{N}{\tau}
$$

where $\mathrm{x}(\mathrm{i})$ is the input signal, $\tau$ is scale and $\mathrm{y}_{\mathrm{j}}^{(\tau)}$ is a signal in scale $\tau$. In general, the signal $\mathrm{y}_{\mathrm{j}}^{(\tau)}$ is the output signal on a scale $\tau$ as the average of a number $\tau$ of the input signal $\mathrm{x}(\mathrm{i})$. Initially, this method was used in conjunction with a sample of entropy to its entropy calculations [9]. In this paper, the coarse-grained procedure was used together with the following entropy measurement to extract the features of lung sounds. 


\subsection{Entropy measurement}

In this paper, we used eight entropy measurement methods to be combined with the coarse-grained procedure. This section explains each entropy measurement method.

\subsubsection{Shannon Entropy}

Shannon entropy (ShEN) is a signal complexity metric. ShEN shows information contain in the signal as expressed in (5).

$$
\operatorname{ShEN}=-\sum_{i=1}^{N} p_{i} \log _{2} p_{i}
$$

where $p i$ is distribution probability of signal, and $i$ is a level of the signal.

\subsubsection{Spectral Entropy}

Spectral entropy (SEN) is ShEN measurement in the frequency domain as expressed as (6).

$$
S E N=-\sum_{f=0}^{f h} p_{f} \log _{2}\left(\frac{1}{p_{f}}\right)
$$

where $P_{\mathrm{f}}$ is the power density of frequency band, and $\mathrm{f}_{\mathrm{i}}$ and $\mathrm{f}_{\mathrm{h}}$ are the frequency limit of the signal. The power of the signal was normalized so that $\sum \mathrm{p}_{\mathrm{n}}=1$.

\subsubsection{Renyi Entropy}

Renyi entropy (REN) is the general form of ShEN [19]. Mathematically REN is expressed as (7).

$$
S_{q}=\frac{1}{1-q} \log _{2}\left(\sum_{i=1}^{N} p_{i}^{q}\right), q \neq 1
$$

where $q=$ order of Renyi entropy. Practically, REN is defined for order $q=2$.

\subsubsection{Wavelet Entropy}

Wavelet Entropy (WE) is calculated from the energy of each sub-band in the wavelet transformation results [20]. WE is expressed as (8).

$$
W E=-\sum_{i<0} p_{i} \ln p_{i}
$$

where $p i$ is the relative wavelet energy obtained from (9).

$$
p_{i}=\frac{E_{i}}{E_{t}}
$$

where Ei is the energy for $\mathrm{i}$-th resolution, and Et is the total energy. The advantages of WE that it is not influenced by noise. WE detects some small changes in non-stationary signals and does not depend on any parameters [20]. In this paper, we used Db2 as mother wavelet and decomposition level = 7, as in [30].

\subsubsection{Approximate Entropy}

Approximate entropy (ApEn) is a signal complexity parameter by measuring the number of occurrences of a signal pattern along the signal [21]. If the sequence of the signal along $\mathrm{N}\{u(i):, 1 \leq i \leq N\}$ is given $m$ to form a vector $X_{i}^{m}$ until $X_{N-m+1}^{m}$ as in (10).

$$
X_{i}^{m}=\{u(i), u(i+1), \ldots, u(i+m-1)\}, \text { for } i=1, \ldots, N-m+1
$$

where $m$ is the length of window which would be compared. For each $i \leq N-m+1$, the defined $C_{i}^{m}(r)$ is $(N-m+1)^{-1}$ multiplied by a number of the the $X_{j}^{m}$ in $r$ from $X_{i}^{m}$. By defining (11)

$$
\emptyset^{\mathrm{m}}(\mathrm{r})=(\mathrm{N}-\mathrm{m}+1)^{-1} \sum_{\mathrm{i}=1}^{\mathrm{N}-\mathrm{m}+1} \ln \mathrm{C}_{\mathrm{i}}^{\mathrm{m}}(\mathrm{r})
$$

where $\ln$ is natural logarithm.

Pincus defined ApEn as in (12) [21].

$$
\operatorname{ApEn}(\mathrm{m}, \mathrm{r})=\lim _{\mathrm{N} \rightarrow \infty}\left[\Phi^{\mathrm{m}}(\mathrm{r})-\Phi^{\mathrm{m}+1}(\mathrm{r})\right]
$$


ApEn was estimated using statistic as (13).

$$
\operatorname{ApEn}(\mathrm{m}, \mathrm{r}, \mathrm{N})=\Phi^{\mathrm{m}}(\mathrm{r})-\Phi^{\mathrm{m}+1}(\mathrm{r})
$$

Commonly ApEn was calculated using $\mathrm{m}=2$ and $\mathrm{r}=0.2 \times$ standard deviation.

\subsubsection{Sample Entropy}

Sample entropy (SampEn) was proposed by Richman and Moorman to overcome the weakness of ApEn [22]. In ApEn, bias is present by self-matches. SampEn is a measure of the probability of a row of $\mathrm{m}$ data that would be the same as other in a series of the signal with a tolerance of $r$, which would remain the same if the row of $m$ data is increased to $m+1$. Mathematically SampEn is expressed as (14).

$$
\operatorname{SampEn}(m, r)=\lim _{N \rightarrow \infty}-\ln \frac{A^{m}(r)}{B^{m}(r)}
$$

where $A^{m}(r)$ is the probability of two sequences will match for a number of $\mathrm{m}+1$ samples within tolerance $\mathrm{r}$. Meanwhile, $B^{m}(r)$ is the probability of two sequences that would match for $m$ number of samples within the tolerance of $r$. In both parameters, self-matches was avoided. Furthermore, by making $B=\{[(N-m-$ 1) $(N-m)] / 2\} B^{m}(r)$ and $A=\{[(N-m-1)(N-m)] / 2\} A^{m}(r)$ so SampEn can be expressed as $(15)$.

$$
\operatorname{SampEn}(\mathrm{m}, \mathrm{r}, \mathrm{N})=-\ln \frac{\mathrm{A}}{\mathrm{B}}
$$

\subsubsection{Permutation Entropy}

Permutation entropy (PE) is the measurement of signal complexity by identifying the probability of code sequence in the signal [23]. PE observes the permutation patterns of the different elements in the signal. It is expressed as (16).

$$
P E=-\sum_{j=1}^{n} p_{j} \log _{2} p_{j}
$$

where $p_{j}$ is the relative frequency of possible pattern and $\mathrm{n}$ is is permutation order, $\mathrm{n} \geq 2$. In this paper, we used $n=6$ as in [11].

\subsubsection{Tsallis Entropy}

Tsallis entropy (TE) is commonly used to describe the physical behavior of a system [24]. TE describes the system with the effect of long-term memory, long-range interaction and multifractal space-time constraint [28]. It is non-extensive in which there are two identical systems, then the number of entropy is not the same as both added together. TE mathematically is expressed as (17).

$$
\mathrm{TE}=\frac{1-\sum_{\mathrm{i}=1}^{\mathrm{W}} \mathrm{p}_{\mathrm{i}}^{\mathrm{q}}}{\mathrm{q}-1}
$$

where $\mathrm{q}$ is non-extensivity order, pi is discrete probability, and $\mathrm{W}$ is the microscopic configuration of the system. In this paper we use the order of non-extensivity $q=2$ proved to produce the highest accuracy in [31].

\section{RESULTS AND DISCUSSION}

\subsection{Measurement Result and Statistics Analysis}

Figure 4 shows the results of the multiscale process using the coarse-grained procedure for the normal bronchial and lung sound crackle sound. In general, the signal shape did not change but the data length becomes $\mathrm{N} / \tau$, with $\mathrm{N}$ is the original data length, and $\tau$ is the scale. The value of each sample on the scale $\tau$ was the average value of $\tau$ sample data from the original data. The coarse-grained procedure reduced the variance of the signal, as the scale $\tau$ increase then the variance will decrease. The decrease in variance will change the entropy measurement value. The change of entropy value was utilized as a feature for pulmonary sound classification. Figure 5 displays the variance as a function of scale $\tau$. 

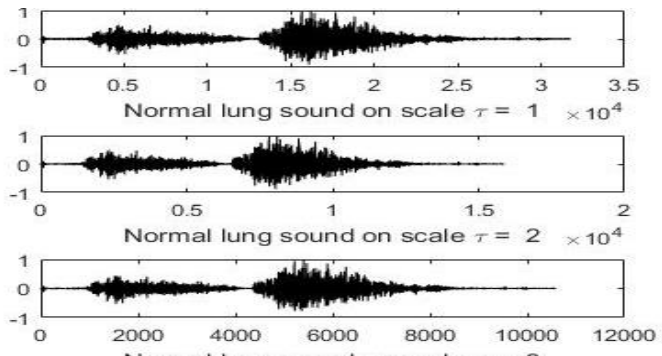

Normal lung sound on scale $\tau=3$

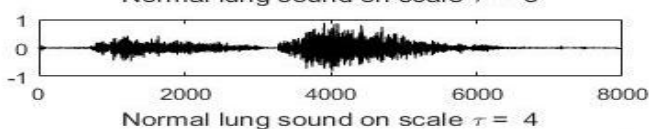

Normal lung sound on scale $\tau=4$

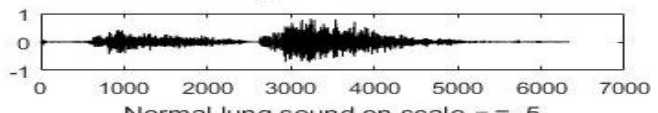

Normal lung sound on scale $\tau=5$

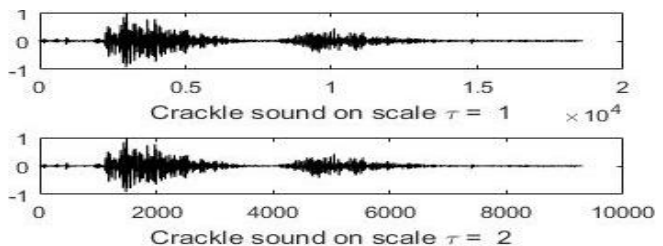

sound on scale $\tau=2$

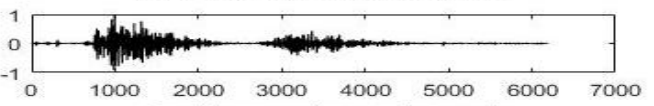

Crackle sound on scale $\tau=3$
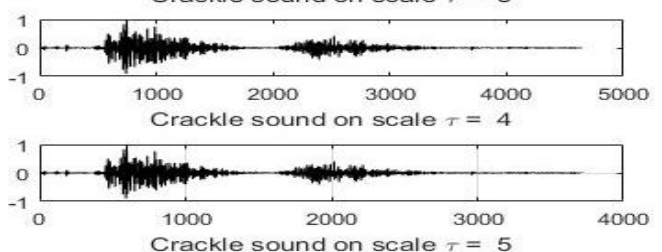

Figure 4. Normal bronchial and crackle lung sound for scale 1 - scale 5

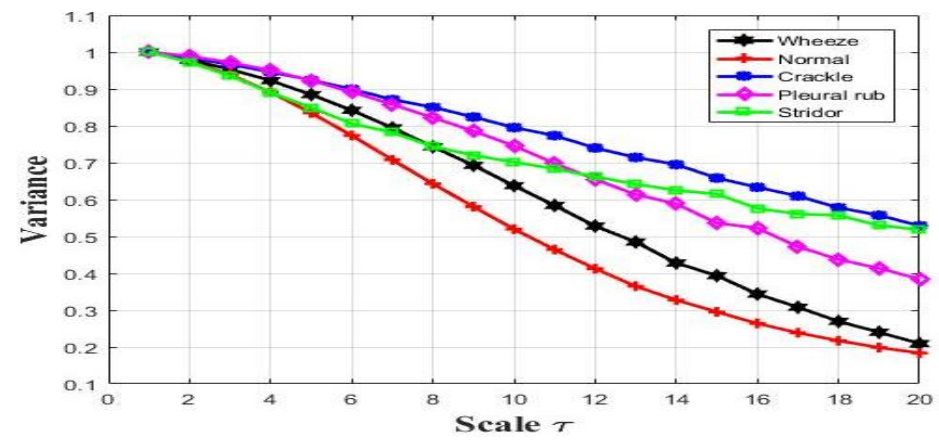

Figure 5. The variance of each class of data

Figure 6 - Figure 13 show the average values of the multiscale entropy measurement results in five classes of lung sounds. The entropy measurement results tended to be low except Tsallis entropy that generated a considerable value but negative value. A small value was influenced by the normalization process so that the average value $=0$ while the variance value became $=1$. We could identify that spectral entropy and wavelet entropy generated relatively distinct patterns between classes. The result suggested that the entropy measurement in the frequency domain produced more distinct characteristics than in the time domain. Figure 10 and Figure 11 display that wheeze sound on the SampEn and ApEn have separated values to other classes. SampEn is an improvement of ApEn that has some self-matching problems [45]. The calculation of both entropies is about the same; hence the resulting entropy also has a similar characteristic.

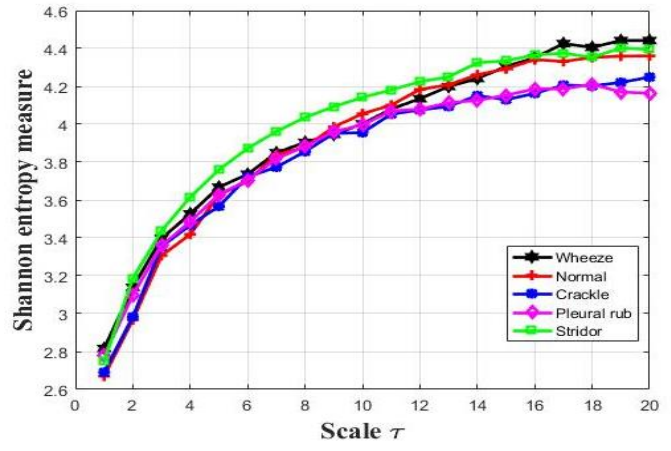

Figure 6. Shannon entropy measurement for five lung sound classes

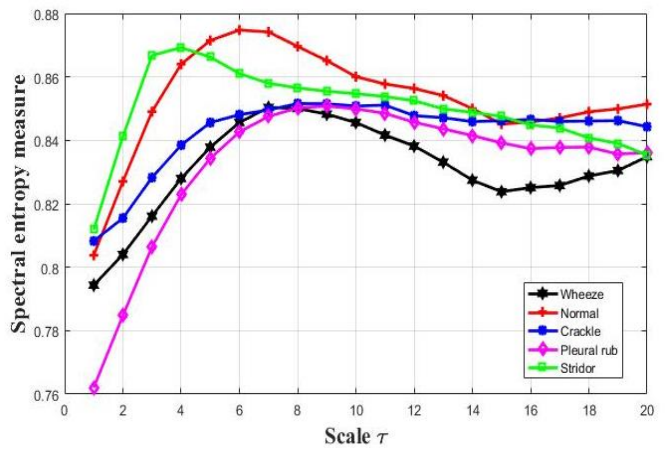

Figure 7. Spectral entropy measurement for five lung sound classes 


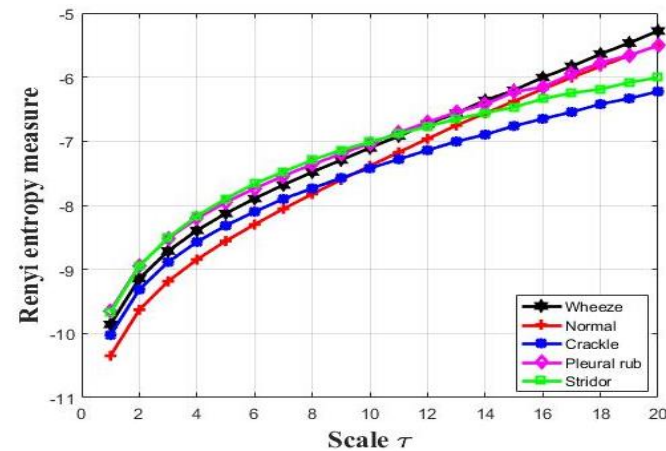

Figure 8. Renyi entropy measurement for five lung sound classes

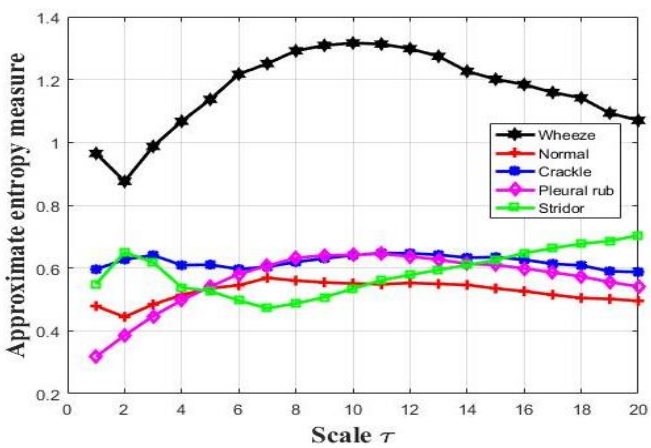

Figure 10. Approximate entropy measurement for five lung sound classes

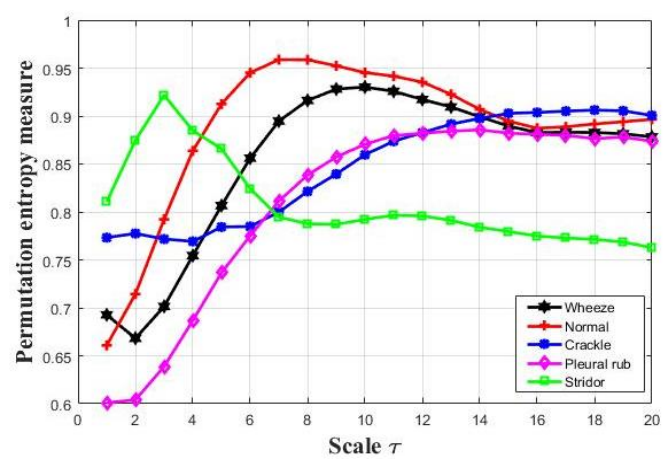

Figure12. Permutation entropy measurement for five lung sound classes

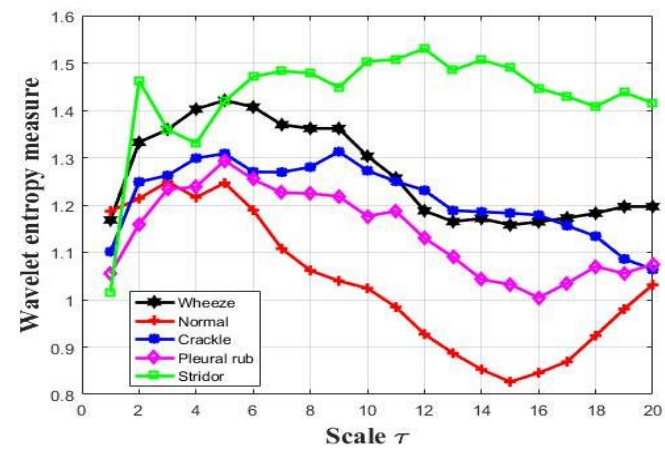

Figure 9. Wavelet entropy measurement for five lung sound classes

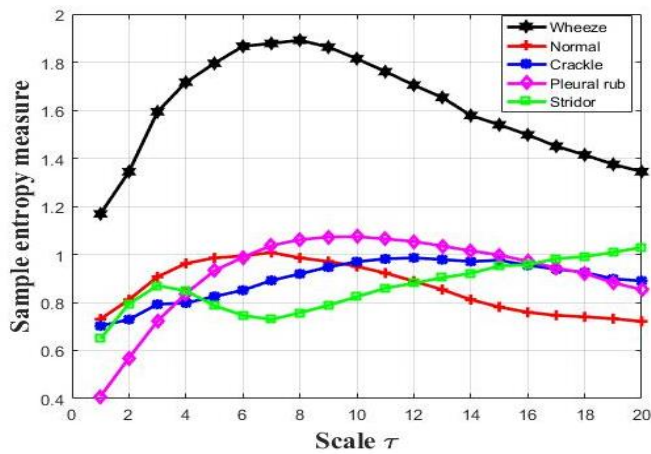

Figure11. Sample entropy measurement for five lung sound classes

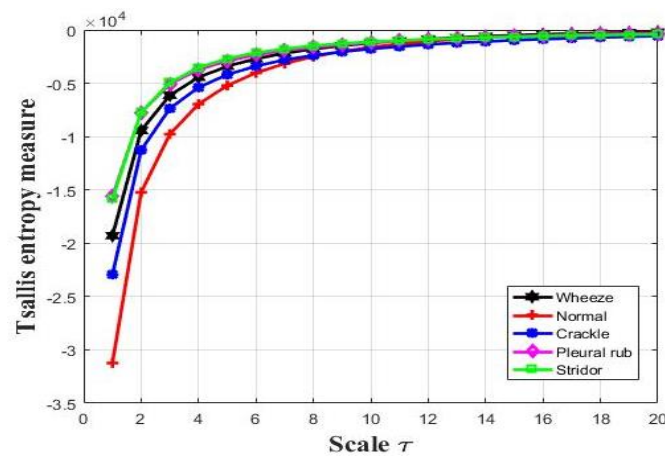

Figure13. Tsallis entropy measurement for five lung sound classes

We used the analysis of variance (ANOVA) statistical test to test the separation between the classes of feature resulting from the multiscale entropy. The performance of multiscale entropy was determined using The ANOVA F-value [32]. A larger F-value indicates a better performance for separating the classes tested compared to the lower one. In this study, F-value was measured on the scale of 1-20, 1-15 scale, and so on to see the changes in the discrimination performance at different scales. Table 2 shows the results ANOVA test.

Table 2 shows that the multiscale sample entropy produced the largest F-value on the scale of 1-20 and the scale of 1-15. For the scale of 1-10 and scale of 1-5, the highest F- value was achieved by Renyi entropy. For the scale of 1 to the scale of 1-4, the highest F- value was achieved by Tsallis entropy. Meanwhile, multiscale Shannon entropy produced the lowest F-value in all conditions.

It was widely perceived that the F-value decreased as the reduction scale except on multiscale Tsallis entropy and multiscale Renyi entropy. In multiscaleTsallis entropy, F-value increased on the scale of 1-15 and then decreased in the next scale. Meanwhile, in multiscale Renyi entropy, F-value of 1-20 scale 
increased to a scale of 1-10 then decreased. This indicated that reducing the scale or reducing the number of features did not always reduce the rate of separation of data but may increase it. This occurred because of the high scale (scale 15 to scale 20) the variance of the signal tended to decrease [10].

Thus the entropy generated was not significantly different from other data. The smallest F- value for all the multiscale entropy was obtained in scale 1 or measurement of entropy in the original signal. This suggested that the multiscale scheme produces a better performance compared to entropy measurement of on a single scale signal only.

Table 2. F-Values from ANOVA Test

\begin{tabular}{|c|c|c|c|c|c|c|c|c|}
\hline \multirow{3}{*}{ MS-Entropy } & \multicolumn{8}{|c|}{ F value } \\
\hline & scale & scale & scale & scale & scale & scale & scale & scale \\
\hline & & & & & & & & \\
\hline Multiscale Tsalli's entropy & 637.19 & 638.20 & 615.42 & 481.38 & 425.38 & 354.12 & 263.91 & 148.62 \\
\hline Multiscale Approximate Entropy & 579.57 & 415.65 & 252.37 & 92.46 & 69.20 & 49.66 & 33.38 & 19.51 \\
\hline Multiscale Wavelet entropy & 400.53 & 322.37 & 150.48 & 41.75 & 32.13 & 24.57 & 15.22 & 10.63 \\
\hline Multiscale Spectral entropy & 371.63 & 322.34 & 287.77 & 248.40 & 210.23 & 151.73 & 81.69 & 31.33 \\
\hline Multiscale Renyi entropy & 360.94 & 442.09 & 620.06 & 512.14 & 434.54 & 340.49 & 233.71 & 118.13 \\
\hline
\end{tabular}

\subsection{Accuracy Testing using Multilayer Perceptro}

To determine the performance of multiscale entropy in the lung sound classification, we used multilayer perceptron (MLP) as a classifier. MLP is one variant of artificial neural networks that have supervised learning properties. Because the MLP was supervised, then we used the N-fold cross-validation $(\mathrm{N}$-fold $\mathrm{CV}$ ) for the training and testing process [33]. Because the number of data in each class is 18 to 22 data, then, the $\mathrm{N}$-fold $\mathrm{CV}$ used $\mathrm{N}=3$ so that each dataset would have $6-8$ data. Table 3 presents its result. Table 3 shows that the multiscale permutation entropy produced the highest accuracy in most of the scales. Multiscale Permutation Entropy (MPE) produced the lower accuracy compared to multiscale sample entropy (MSE) on the scale of 1-5 and scale of 1-4. PE had several advantages compared with SampEn: PE calculated the permutation of the sequence pattern of the signal, while the SampEn calculated the number of the same patterns of the signal with a certain tolerance. This implies that the SampEn calculation takes longer computation time rather than the PE. Similarities between those two patterns were determined by several parameters, such as the n-order permutation on PE, meanwhile, in SampEn, they were determined by the similarities tolerance $r$ and length of pattern $m$.

Table 3. Accuracy (\%) for various multiscale entropies using three-fold cross-validation

\begin{tabular}{|c|c|c|c|c|c|c|c|c|}
\hline MS Entropy & $\begin{array}{c}\text { scale } \\
1-20 \\
\end{array}$ & $\begin{array}{c}\text { scale } \\
1-15 \\
\end{array}$ & $\begin{array}{c}\text { Scale } \\
\text { scale } \\
1-10\end{array}$ & $\begin{array}{c}\text { for ent } \\
\text { scale } \\
1-5\end{array}$ & $\begin{array}{c}\text { oy meas } \\
\text { scale } \\
1-4\end{array}$ & $\begin{array}{c}\text { ment } \\
\text { scale } \\
1-3\end{array}$ & $\begin{array}{c}\text { scale } \\
1-2\end{array}$ & $\begin{array}{c}\text { scale } \\
1\end{array}$ \\
\hline Multiscale Tsalli's entropy & 88.89 & 89.9 & 91.92 & 79.8 & 73.74 & 72.73 & 70.71 & 69.7 \\
\hline Multiscale Wavelet entropy & 74.75 & 68.69 & 67.68 & 70.71 & 70.71 & 71.72 & 66.67 & 43.43 \\
\hline Multiscale Spectral entropy & 93.94 & 95.96 & 95.96 & 90.91 & 89.9 & 80.81 & 75.76 & 49.49 \\
\hline Multiscale Renyi entropy & 88.89 & 90.91 & 93.94 & 78.79 & 70.71 & 74.75 & 69.7 & 68.69 \\
\hline
\end{tabular}


Table 4 summarizes the best results for each of multiscale entropy including a scale that produces the highest accuracy, the accuracy of the single scale and average computation time for a single data. Tests were performed on a PC with the specification of Intel (R) Core (TM) i3-3220 CPU @ 3.30GHz, 4 GB of RAM.

Table 4 displays that the computational time for the overall technique was acceptable except for the multiscale approximate entropy. It took a very long time, so it was less reliable used in real applications. A self-matching process caused long computation time occurred in ApEn computation.

Table 4. Scale for the highest accuracy and computation time

\begin{tabular}{lcccc}
\hline \multicolumn{1}{c}{ Multiscale Entropy } & Best scale & $\begin{array}{c}\text { Highest } \\
\text { Accuracy }(\%)\end{array}$ & $\begin{array}{c}\text { Accuracy for } \\
\text { single scale }(\%)\end{array}$ & $\begin{array}{c}\text { Average computation } \\
\text { time (s) }\end{array}$ \\
\hline Multiscale Sample entropy & $1-5$ & 86.87 & 50.51 & 27.69 \\
Multiscale Tsalli's entropy & $1-10$ & 91.92 & 69.7 & 0.69 \\
Multiscale Approximate Entropy & $1-15$ & 88.89 & 51.52 & 2087.47 \\
Multiscale Permutation entropy & $1-10$ & 97.98 & 59.6 & 1.61 \\
Multiscale Wavelet entropy & $1-20$ & 74.75 & 43.43 & 1.00 \\
Multiscale Spectral entropy & $1-10$ & 95.96 & 49.49 & 0.93 \\
Multiscale Renyi entropy & $1-10$ & 93.94 & 68.69 & 0.70 \\
Mutliscale Shannon entropy & $1-15$ & 84.85 & 37.37 & 0.84 \\
\hline
\end{tabular}

Table 4 shows that the MPE produced the highest accuracy on the scale 1-10 with an accuracy of $97.98 \%$. Some multiscale entropy only used scale 1-5 to produce the highest accuracy, but still below the MPE. Hence, it was not the best choice. Regarding computing time, the MPE required a reasonable computing time, i.e., $1.62 \mathrm{~s}$ for the single data. Meanwhile, multiscale ApEn took a long computational time. This was caused by the calculation of the number of similar patterns of data carried along the sequence of the signals and the emergence of self-matching. Multiscale SampEn (MSE) overcame the weakness multiscale ApEn, so MSE required shorter computation time than the multiscale ApEn.

Multiscale SEN produced the highest accuracy after multiscale PE as some signal differences between the classes could be seen clearly in the spectral frequency. However, multiscale WE could not produce a quite high accuracy for being influenced by the mother wavelet selection, the sampling frequency and the level of decomposition. These factors would affect the formed sub-band. The selection of DB2 and decomposition level 7 did not produce a high accuracy. Appropriate Wavelet filter and the level of decomposition selection will be the next research.

ShEN, REN, and TE have a similar form. ShEN is a special case of REN and TE. ShEN is REN or TE with order $q=1$. The selection of order $q$ in REN and TE will produce the different accuracy. The use of TE with a different order for lung sound analysis was presented in [31]. The result showed that nonextensivity order $\mathrm{q}=2$ produces the best feature for the lung sound analysis.

The entropy measurement in the multiscale scheme increased lung sound classification accuracy compared with the single scale schemes. Several previous studies used only one entropy values coupled with the other features of the lung sound classification. Sample entropy was combined with skewness, kurtosis, and lacunarity reported by Mondal et al [34]. The resulting accuracy was $92.86 \%$ for normal and abnormal lung sound. Meanwhile, kurtosis, mean crossing irregularity and Renyi entropy used in [35]. The results obtained the accuracy of $95.1 \%$ for the training data and $93.5 \%$ for testing data. Tsallis entropy and 25 other characteristics used by Morillo et al. for lung sound from congestive obstructive pulmonary disease (COPD) patients [36]. Meanwhile, Jin et al. used sample entropy as the features of lung sound extracted from the short-time Fourier transform (STFT) [37].

The same entropies as in this paper were used in [38]. Single entropy only produced $69.7 \%$ of accuracy using TE. Composite of seven entropies increased the accuracy became $94.9 \%$. Computation of seven entropies was more complex than one entropy with the multiscale process. Compared with this paper, 94.9\% was achieved using seven features while $97.9 \%$ was achieved using ten features.

Overall the studies were conducted on single scale signal. We could see that entropy still needed other features for lung sound classification. While on the multiscale entropy, we only used only entropy that calculated on a signal with a different scale. Although it was not directly comparable, multiscale entropy provided more promising results. Direct comparisons with the same dataset may be made on the research in the future. 
Some multiscale entropies were proposed by some researchers for solving various cases in previous studies. Multiscale sample entropy which was more known as multiscale entropy (MSE) was proposed by Costa et al [9]. Multiscale permutation entropy was used for quantifying signal complexity proposed by Azis and Arif [11]. In this paper, we introduced several multiscale entropies for signal complexity measurement. For example multiscale Tsallis entropy, multiscale Renyi entropy, multiscale spectral entropy and multiscale wavelet entropy. Multiscale entropy provides better performance for lung sound analysis compared to entropy in single scale [38].

\section{CONCLUSION}

Lung sound is one of the biological signals which have information about the health of the respiratory system. To reduce subjectivity in the evaluation of lung sounds $t$ a variety of digital signal processing techniques for automatic lung sound classification have been developing. One of the methods used in lung sound signal processing is the entropy measurement method. In this research, we compared multiscale entropy with some different entropy measurement techniques for lung sound feature extraction. The results showed that the multiscale permutation entropy produced the highest accuracy of $97.98 \%$ with the scale of 1-10. These results are much higher than using Permutation entropy individually on the overall signal. Some entropy measurement parameters that could be altered to change such as Tsallis entropy or Renyi entropy for a different order of q. Performance of multiscale entropy with various entropy parameters can be examined in subsequent research.

\section{ACKNOWLEDGEMENTS}

This work has been financially supported by Ministry of Research, Technology, and Higher Education of Republic of Indonesia under Penelitian Disertasi Doktor Scheme no: 014/PNLT3/PPM/2018.

\section{REFERENCES}

[1] Pasterkamp H, Kraman SS, Wodicka GR. Respiratory Sounds. Am J Respir Crit Care Med. 1997;156(3):974-987.

[2] Spieth PM, Zhang H. Analyzing Lung Crackle Sounds: Stethoscopes And Beyond. Intensive Care Medicine. 2011;37(8):1238-9.

[3] Rizal A, Hidayat R, Nugroho HA. Multiscale Hjorth Descriptor for Lung Sound Classification. International Conference on Science and Technology 2015. Yogyakarta. 2015; 1:160008.

[4] Chen X, Shao J, Long Y, Que C, Zhang J, Fang J. Identification of Velcro Rales Based on Hilbert-Huang Transform. Phys A Stat Mech its Appl. 2014; 401(8):34-44.

[5] Gnitecki J, Moussavi Z, Pasterkamp H. Classification of lung sounds during bronchial provocation using waveform fractal dimensions. Annual International Conference of the IEEE Engineering in Medicine and Biology Society. 2004; 3844-3847.

[6] Mayorga P, Druzgalski C, Gonzalez OH, Lopez HS. Modified Classification of Normal Lung Sounds Applying Quantile Vectors. Proceedings of the Annual International Conference of the IEEE Engineering in Medicine and Biology Society, EMBS. 2012; 4262-4264.

[7] Chen C, Huang W, Tan T, Chang C-C, Chang Y. Using K-Nearest Neighbor Classification to Diagnose Abnormal Lung Sounds. Sensors . 2015 Jun 4;15(6):13132-58.

[8] Kandaswamy A, Kumar CS, Ramanathan RP, Jayaraman S, Malmurugan N. Neural classification of lung sounds using wavelet coefficients. Comput Biol Med. 2004;34(6):523-537.

[9] Costa M, Goldberger AL, Peng CK. Multiscale Entropy Analysis of Biological Signals. Phys Rev E - Stat Nonlinear, Soft Matter Phys. 2005;71:1-18.

[10] Humeau-heurtier A. The Multiscale Entropy Algorithm and Its Variants: A Review. Entropy. 2015;17:3110-3123.

[11] Aziz W, Arif M. Multiscale Permutation Entropy of Physiological Time Series. In: 9th International Multitopic Conference, IEEE INMIC 2005. Karachi 2005;1-6.

[12] Wang G-J, Xie C, Han F. Multi-Scale Approximate Entropy Analysis of Foreign Exchange Markets Efficiency. Syst Eng Procedia. 2012;3(2011):201-208.

[13] Xie H, Chen W, He W, Liu H. Complexity analysis of the biomedical signal using fuzzy entropy measurement. Appl Soft Comput. 2011;11(2):2871-2879.

[14] Charleston-Villalobos S, Albuerne-Sanchez L, Gonzalez-Camarena R, Mejia-Avila M, Carrillo-Rodriguez G, Aljama-Corrales T. Linear and Nonlinear Analysis of Base Lung Sound in Extrinsic Allergic Alveolitis Patients in Comparison to Healthy Subjects. Methods Inf Med. 2013;52(3):266-76.

[15] Wu N, Guo Y, Wei Y, Fan S, Li X. Design of New Transformer Protection Device Based on Wavelet Energy Entropy-Neural Network Theory and. TELKOMNIKA. 2013;11(11):6746-6754.

[16] Xu X, Wang S, Liu J, Wu Z. Fan Fault Diagnosis Based on Wavelet Packet and Sample Entropy. TELKOMNIKA. 2013;11(6):3451-3462.

[17] Dong Z, Tian X, Zeng J. Mechanical Fault Diagnosis Based on LMD- Approximate Entropy and LSSVM. TELKOMNIKA. 2013;11(2):803-808. 
[18] Shannon CE. A Mathematical Theory of Communication. Bell Syst Tech J. 1948;27(3):379-423.

[19] Renyi A. On Measures of Entropy and Information. Proceedings of the fourth Berkeley Symposium on Mathematics, Statistics and Probability. 1960; 547-561.

[20] Rosso OA, Blanco S, Yordanova J, Kolev V, Figliola A, Schu M, et al. Wavelet entropy : a new tool for analysis of short duration brain electrical signals. J Neurosci Methods. 2001;105:65-75.

[21] Pincus SM. Approximate Entropy as a Measure of System Complexity. Proc NatlAcad Sci USA. 1991;88(March):2297-2301.

[22] Richman JS, Moorman JR. Physiological time-series analysis using approximate entropy and sample entropy. Am J Physiol Heart Circ Physiol. 2000;278:H2039-2049.

[23] Bandt C, Pompe B. Permutation Entropy: A Natural Complexity Measure for Time Series. Phys Rev Lett. 2002;88(17):174102.

[24] Tsallis C. Possible Generalization of Boltzman-Gibbs Statistics. J Stat Phys. 1988;52(1/2):479-87.

[25] The R.A.L.E Repository. Available from: http://www.rale.ca/Repository.htm

[26] The Auscultation Assistant - Breath Sounds. Available from: http://www.med.ucla.edu/ wilkes/lungintro.htm

[27] Wilkins RL, Hodgkin JE, Lopez B. Lung Sounds: A Practical Guide with Audio CD. 2nd ed. Maryland Heights, Missouri: Mosby; 1996.

[28] Bohadana A, Izbicki G, Kraman SS. Fundamentals of lung auscultation. N Engl J Med . 2014; 370(8):744-751.

[29] Reichert S, Gass R, Brandt C, Andrès E. Analysis of Respiratory Sounds : State of the Art. Clin Med Circ Respirat Pulm Med. 2008;2008(2):45-58.

[30] Rizal A, Hidayat R, Nugroho HA. Multiresolution Modified Grey Level Difference for Respiratory Sound Classification. The 2016 Advance Research in Engineering and Information Technology International Conference (AREITIC). Bandung, Indonesia; 2016;1-5.

[31] Rizal A, Hidayat R, Nugroho HA. Pulmonary Crackle Feature Extraction using Tsallis Entropy for Automatic Lung Sound Classification. The 1st 2016 International Conference on Biomedical Engineering (iBioMed). Yogyakarta; 2016; 1: 8-11.

[32] Acharya UR, Fujita H, Sudarshan VK, Bhat S, Koh JEW. Application of entropies for automated diagnosis of epilepsy using EEG signals : A review. Knowledge-Based Syst. 2015;88:85-96.

[33] Andersen T, Martinez T. Cross Validation and MLP Architecture Selection. IJCNN'99 International Joint Conference on Neural Networks Proceedings. 1999; 1614-1619.

[34] Mondal A, Bhattacharya P, Saha G. Detection of Lungs Status using Morphological Complexities of Respiratory Sounds. Sci World J. 2014;2014:1829-1838.

[35] Aydore S, Sen I, Kahya YP, Mihcak MK. Classification of Respiratory Signals by Linear Analysis. Annual International Conference of the IEEE Engineering in Medicine and Biology Society. 2009; 2617-2620.

[36] Morillo DS, Jiménez AL, Moreno SA. Computer-Aided Diagnosis of Pneumonia in Patients With Chronic Obstructive Pulmonary Disease. J Am Med Informatics Assoc. 2013;20(e1):e111--e117.

[37] Jin F, Sattar F, Goh DYT. Automatic Wheeze Detection using Histograms of Sample Entropy. Annual International Conference of the IEEE Engineering in Medicine and Biology Society IEEE Engineering in Medicine and Biology Society. 2008: 1890-1893.

[38] Rizal A, Hidayat R, Nugroho HA. Entropy Measurement as Features Extraction in Automatic Lung Sound Classification. 2017 International Conference on Control, Electronics, Renewable Energy and Communications (ICCREC). Yogyakarta. 2017;3: 93-97. 